\title{
Toward Accessible Hazard Mapping: Tactile Risk Maps and Disaster Preparedness
}

\author{
Harrison Cole ${ }^{\mathrm{a}}$ \\ a The Pennsylvania State University
}

Keywords: Tactile maps, risk, natural hazards, emergency management, accessibility

\begin{abstract}
:
The near future of our planet under climate change is predicted to be characterized by increasingly frequent and severe natural hazards of all kinds. Understanding the potential spatial extent and impacts of these hazards is a critical component of creating effective emergency management plans, not just on the part of emergency managers and relief organizations, but also for those who may reside in areas vulnerable to disaster. Often, information about disaster risk is communicated using maps, such as in the case of storm surge maps, evacuation zone maps or wildfire extent maps. While generally helpful, these maps are of limited use to those with low vision or blindness, and the information is rarely, if ever, distributed in accessible formats. Furthermore, in contrast to wayfinding maps, this type of information does not lend itself to being translated into a set of instructions to be read aloud by a test-to-speech device. Instead, risk maps are useful because they allow users to identify and assess spatial relationships between many features on the maps- a key process that would be muddled if the maps were to be verbally described, and thus a process that is currently inaccessible to those who cannot see the maps. As people with visual impairments are especially vulnerable in disaster scenarios, working towards the universal accessibility of spatial information is imperative for inclusive disaster preparedness and hazard mitigation.
\end{abstract}

Many challenges exist in creating maps for people with visual impairments. The most obvious is the fact that a visual medium cannot be used by someone who is unable to see. It is important to note that "visual impairment" covers a broad spectrum of conditions from pronounced myopia to complete blindness. Here, I will be limiting my topical scope to people with total or near-total blindness. Tactile maps are the tool of choice for this population. However, it is generally harder to distinguish between similar patterns using touch than using sight, so features on a tactile map need to be unique and unambiguous. This makes it more challenging to communicate information typically represented using color gradients or transparency. Additionally, distributing spatial information in tactile form poses another layer of complications. There are several options for creating tactile maps, from embossed paper to 3D printing, and each come with their own advantages and drawbacks. Cost, speed and detail all vary among these media, and thus each potentially impact the user's perception of risk.

In my talk, I will be examining these challenges as they intersect with research on cartographic risk visualization, emergency management, and tactile maps in general. First, I will briefly discuss the state of risk visualization in the context of emergency management, focusing on preparedness. Then, I will give a general overview of the extant research on tactile maps with particular attention paid to their influence on spatial cognition. Next, I will examine how the considerations particular to risk map creation are affected by the potentials and limitations of tactile maps. Finally, I will outline a research agenda for creating and distributing accessible maps for emergency management. Using visual impairment and tactile maps as starting points, I will identify further aspects of emergency management that urgently require greater attention to accessibility, and how cartographic research and technology can help bridge those gaps.

Planning for natural hazards is an essential step in mitigating their impacts on communities, and that process includes individual citizens making their own plans for evacuation, finding shelter or securing food, water or medicine. This is especially important for people with disabilities, as resources deployed after a disaster may fail to accommodate their particular needs, and processes such as evacuating a building or house can take substantially longer than average. Planning for these scenarios is made all the more difficult for people with visual impairments, as conventional maps are unhelpful, and environmental awareness is limited. Thus, empowering people with visual impairments to make emergency plans for themselves and contribute to planning for their community as a whole can help build autonomy and self-confidence and ultimately ensure that disaster plans truly account for everyone. 